\title{
Convective Heat Transfer and Infrared Thermography (IRTh)
}

\author{
J.M. Buchlin \\ von Karman Institute for Fluid Dynamics \\ Chaussée de Waterloo 72, B-1640, Rhodes-Saint-Genèse, Belgium
}

Email:buchlin@vki.ac.be

(Received February 25, 2009; accepted March 28, 2009)

\begin{abstract}
The paper deals with the application of the infrared thermography to the determination of the convective heat transfer coefficient in complex flow configurations. The fundamental principles upon which the IRTh relies are reviewed. The different methods developed to evaluate the heat exchange are described and illustrated through applications to the aerospace and aeronautical field as well as to the industrial processes.
\end{abstract}

Keywords: Thermometry, Thermal radiation, Aerothermal flows, Multijets, Ribs, Fibre spinning.

\section{NOMENCLATURE}

a Wire radius $(\mathrm{m})$

$b \quad$ Effusivity $\left(\mathrm{Jm}^{-2} \mathrm{~K}^{-1} \mathrm{~s}^{-0.5}\right)$

$d \quad$ Hole diameter $(\mathrm{m})$

$f \quad$ Spatial frequency $\left(\mathrm{m}^{-1}\right)$

$h \quad$ Heat transfer coefficient $\left(\mathrm{W} \mathrm{m}^{-2} \mathrm{~K}^{-1}\right)$

$H \quad$ Rib height (m)

$\ell, L \quad$ Length (m)

$M \quad$ Emittance $\left(\left(\mathrm{W} \mathrm{m}^{-2}\right)\right.$

$\mathrm{Nu} \quad$ Nusselt number

$P \quad$ Rib pitch (m)

$q \quad$ Heat flux $\left(\mathrm{Wm}^{-2}\right)$

Re Reynolds number

$S \quad$ Hydraulic diameter (m)

$t \quad$ Time (s)

$T \quad$ Temperature $\left({ }^{\circ} \mathrm{C}\right.$ or $\left.\mathrm{K}\right)$

$U \quad$ Velocity $\left(\mathrm{ms}^{-1}\right)$

$x, y \quad$ Coordinates $(\mathrm{m})$

\section{INTRODUCTION}

The energy conversion and energy control require a good knowledge of the heat exchange mechanisms which govern the thermal behavior and therefore fix the efficiency of energetic systems. The flows and the fluids present in such systems may be of so complex nature that the accurate determination of heat flux demands the implementation of acute measurement techniques.

\author{
Greek letters \\ $\alpha \quad$ Incidence $\left({ }^{\circ}\right)$ \\ $\beta \quad$ Surface porosity \\ $\varepsilon \quad$ Emissivity \\ $\lambda \quad$ Wavelength (m) \\ $v \quad$ Kinematic viscosity $\left(\mathrm{m}^{2} \mathrm{~s}^{-1}\right)$ \\ $\xi \quad$ Curvature factor \\ $\sigma \quad$ Stefan-Boltzmann constant $\left(\mathrm{Wm}^{-2} \mathrm{~K}^{-4}\right)$ \\ Subscripts \\ a Air \\ amb Ambient \\ d Detector \\ f Focal \\ fl $\quad$ Fluid \\ max Maximum \\ s $\quad$ Surface \\ Upperscripts \\ Black body
}

Among the non-intrusive techniques, the infrared thermography (IRTh) has taken a prominent place in research laboratories. It has been significantly developed the two last decades. Taking profit of the technological progresses made in the realization of radiometers, the IRTh offers nowadays the possibility of fast imaging and appears as a powerful quantitative tool to obtain the mapping of temperature and subsequently the heat exchange between a fluid and a solid surface. 
The paper emphasizes the potential of the infrared thermography to deals with complex thermo-fluid problems. In a first section, the basic principles on which IRTh relies are reviewed. The next section presents the methods, which allow the determination of the convective heat transfer coefficient from the measurement of the temperature field. The last section describes some illustrative IRTh application where the convective heat exchange is the key parameter.

\section{BASIC PRINCIPLe OF THE IRTH}

As the temperature of a body, $T$, exceeds the absolute zero, the molecular agitation in the matter results in an electromagnetic radiation. The energy released within this radiation increases as the body temperature rises. The energy content of the emission is also related to the wavelength of the radiation (see Eq. (1))

Depending on the nature of the material and the heating conditions, the emitted thermal radiation can spread continuously over a large range of wavelengths $\lambda$ (continuous spectrum) or can exhibit only distinct bands (discrete spectrum). The electromagnetic radiation that carries thermal energy is confined to the range $0.1 \leq \lambda \leq 1000 \mu \mathrm{m}$ in which one can essentially identify the narrowness of the visible spectrum $(0.4 \mu \mathrm{m}$ to $0.8 \mu \mathrm{m})$ and the infrared spectrum $(0.75 \mu \mathrm{m}$ to 1000 $\mu \mathrm{m}$ ) that is conventionally split into near (from $0.75 \mu \mathrm{m}$ to $1.5 \mu \mathrm{m}$ ), mean (from $1.5 \mu \mathrm{m}$ to $20 \mu \mathrm{m}$ ) and far $(20 \mu \mathrm{m}$ to $1000 \mu \mathrm{m})$ infrared. The maximum heat flux emitted by thermal radiation is coming from an ideal surface called black body. The spectral emissive power of black body, $M_{\lambda}^{o}$, is expressed by the Planck's law, a graphical representation of which is drawn in Fig. 1.

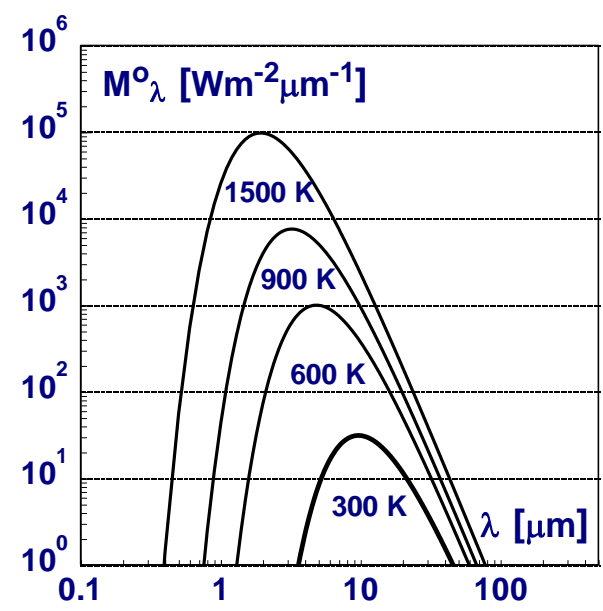

Fig. 1. Spectral emissive power of black body

The total emissive power (or radiant exitance) of black body, $M^{o}$, over all the spectrum is given by the StefanBoltzmann law:

$$
M^{o}=\int_{0}^{\infty} M_{\lambda}^{o} \mathrm{~d} \lambda=\sigma T^{4}
$$

where $\sigma=5,67 \quad 10^{-8} \quad \mathrm{Wm}^{-2} \mathrm{~K}^{-4}$. However, at equal temperature real bodies radiate less thermal energy than black body. Their radiant exitance depends on their emissivity factor, $\varepsilon(<1)$ :

$$
M(T)=\varepsilon M^{o}(T)=\varepsilon \sigma T^{4}
$$

Therefore, the principle of the infrared thermo-graphy consists in capturing the infrared radiation emitted by a surface with a radiometer and transforming this measure in a signal, which once processed leads to the determination of the temperature.

The main difficulty of such a measurement comes from the reflectance of the surface scanned by the IR radiometer. If a surface is characterized by a low emissivity, in accordance with the Kirchhoff's law and energy balance, it will reflect an infrared energetic content emitted by the ambient surrounding at temperature $T_{a m b}$. As a result, the infrared receptor will receive a radiosity quantity from the surface, $E$, containing two contributions:

$E=\varepsilon M^{o}(T)+(1-\varepsilon) M^{o}\left(T_{a m b}\right)$

The second term of the right hand side of Eq. (3) fouls the measurement of $T$. As it is generally difficult to evaluate this term, one will minimize it in providing the surface with a thin coating of a high emissivity in infrared (e.g. paints, varnishes, lampblack, $\varepsilon \approx 0.96$ ).

Most often the model to be scanned by the radiometer is located in a test section fitted with windows. These optical accesses should have a good transmittance in the infrared spectrum. The good candidates are sapphire, germanium and zinc selenide. Nevertheless, due to absorption phenomenon, these windows will yield signal attenuation. This is why it is always recommended to calibrate the measurement IR chain in the experimental configuration. This can easily be done by using thermocouples flush mounted on the model, and correlating the IR radiometer output (generally expressed in Intensity Unit, IU) to the measured temperature.

The infrared radiometer is a camera. It is composed of an optical unit, which collects and focuses the thermal radiation beam on a photovoltaic detector maintained at very low temperature, generally by a liquefied gas ( e.g. nitrogen at $77 \mathrm{~K}$ ) or a cryogenic machine based on the Stirling cycle, to increase its detectivity (Gaussorgues, 1989). In the case of a single detector, the raster is performed by mechanical scanning leading to a twodimensional thermogram, which constitutes the IR picture of the surface. If the detector is a mosaic of sensible elements, the scanning is made electronically. Each pixel of the numerical image is characterized by a grey-level (IU) which corresponds to a temperature value.

Most of the IR cameras work between $3 \mu \mathrm{m}$ and $12 \mu \mathrm{m}$. In this range of wavelength, the atmosphere owns several spectral bands with high IR transmittance. The IR cameras are characterized by thermal and spatial resolution.

The smallest temperature difference which can be discriminated in a thermal field corresponds to the signal to noise ratio equal to 1 . This Noise Equivalent 
Temperature Difference (NETD) may vary from $0.02^{\circ} \mathrm{C}$ to $0.2^{\circ} \mathrm{C}$ according to the camera technology.

The smallest thermal area which can be discriminated by the camera is the Instantaneous Projected Area (IPA) of the detector on the surface of the tested object. This quantity depends on the size of the detector, $\ell_{\mathrm{d}}$, the focal of the reception optics, $\ell_{\mathrm{f}}$, and the standoff distance between the radiometer and the model, $\ell$ :

$$
I P A=\left(\frac{\ell_{d}}{\ell_{f}}\right)^{2} \ell^{2}
$$

As a rule of thumb it can be claimed that a thermal area will be correctly resolved if its size is greater than 5 IPA. Below this limit, signal attenuation appears leading to an underestimation of the real surface temperature.

The same if the surface displays a cyclic temperature variation. The measure of the thermal field can be corrupted if the spatial frequency, $f_{s}$, of this distribution is too high. The ratio of the measured temperature amplitude to the real one is expressed by the Modulation Transfer Function (MTF):

$$
M T F=\frac{\Delta T_{\text {meas }}}{\Delta T_{\text {real }}}=\exp \left[-\left(f / f_{\text {max }}\right)^{2}\right]
$$

In Eq. (5), $f_{\max }$ is a constant of the camera. In order to get the MTF as close as possible to 1, the IPA must be smaller than the span of a thermal cycle, otherwise a correction should be applied (see paragraph concerning the compression ramp).

\section{DETERMination OF THE CONVECTIVE Heat Transfer CoefFicient With IRTH}

The determination of the heat transfer coefficient by convection requires the knowledge of the wall temperature. The measurement can be achieved either during a transient regime or at steady state. The timedependent measurement of the temperature is generally assigned to the so-called passive method while the steady state measurement refers to the active method.

The application of the passive method involves the resolution of the heat equation in function of time and space. The surface temperature distribution, $T_{s}(t, x, y)$, measured by IRTh is used as boundary conditions. In the case of a one-dimensional thermal behavior through the model thickness and constant convective heat transfer coefficient, $h$, the solution of the thermal problem is:

$$
\frac{T_{s}(t, x, y)-T_{s o}(x, y)}{T_{f l}-T_{s o}(x, y)}=1-e^{\frac{h^{2} \cdot t}{b^{2}}} \operatorname{erfc}\left(\frac{h}{b} \sqrt{t}\right)
$$

In Eq. (6), $b$ is the effusivity of the model. According to Eq. (6), the knowledge of the temperature field at the initial condition and at any time $t$ provides the distribution of $h$.
The active method relies essentially on the Newton's relation (Eq. (7)). The wall heat flux, $q_{s}$, is adjusted and controlled (Joule effect, Peltier effect, Laser heating, etc.). The measurement of $T_{s}(x, y)$ at steady state leads straightforwardly to:

$h(x, y)=\frac{q_{s, c v}}{\left(T_{s}(x, y)-T_{\text {fluid }}\right)}$

In Eq. (7) $q_{s, c v}$ is the true heat removed by convection.

When the surface on which the convection develops is not optically accessible, an inverse method has to be applied. The procedure consists in measuring the thermal field on the faces which can be scanned by the IR camera and using these data as boundary conditions to solve a heat condition problem. Such a approach requires also the application of an optimization method to retrieve accurately the thermal data on the masked convective surface (see hereunder the study of the flame impact).

\section{APPLICATIONS OF THE IRTH}

\subsection{High Enthalpy Flows}

The IRTh passive method is suitable to study flows at high enthalpy as such encountered during the reentry of space vehicles in atmosphere. The significant heating is here produced by the fluid friction at the wall. The generic configuration of such flows, which has been studied at the VKI, is the hypersonic compression ramp (Simeonides et al. 1990, 1993). A schematic is shown in Fig. 2a. A wedge is placed in a flow at Mach number equal to 6 . The ramp is composed of a flat plate made out glass ceramics coated with a black paint at high emissivity. The thermogram shown in Fig. $2 b$ indicates that the thermal field exhibits streamwise striations with a well-defined spatial frequency (direction OY). This IR signature, which compares well with visualization obtained by sublimation technique, results from the formation of counter-rotating Görtler vortices. This flow induces a streamwise variation of the heat flux as plotted in Fig. 3. Since the analysis indicates that this striation pattern has a wavelength of about $3 \mathrm{~mm}$ what corresponds to a main spatial frequency of $0.33 \mathrm{mrad}^{-1}$, one may anticipate a degradation of the measure due to a lack of spatial resolution.

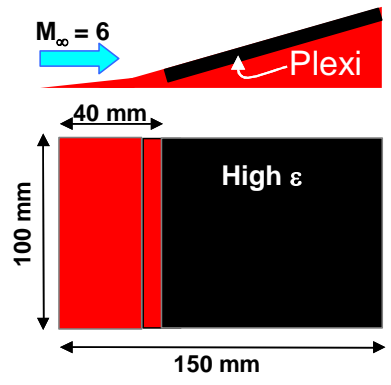

(a)

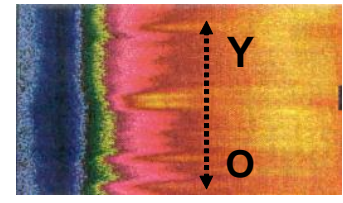

(b)
Fig. 2. Hypersonic compression ramp

After application of the radiometer MTF to the Fast Fourier Transform of the data, one gets from inversion the corrected temperature field: 
J.M. Buchlin / JAFM, Vol. 3, No. 1, pp. 55-62, 2010.

$$
T_{\text {corrected }}=F T^{-1}\left[\frac{F T\left(T_{\text {measured }}\right)}{M T F}\right]
$$

The reajusted heat flux distribution obtained from these new data is plotted for sake of comparison in Fig. 3.

The correction proved to be rather importante $( \pm 15 \%$ with respect to the mean). It could be readily avoided by using for instance an afocal $3 \mathrm{X}$ telescope.

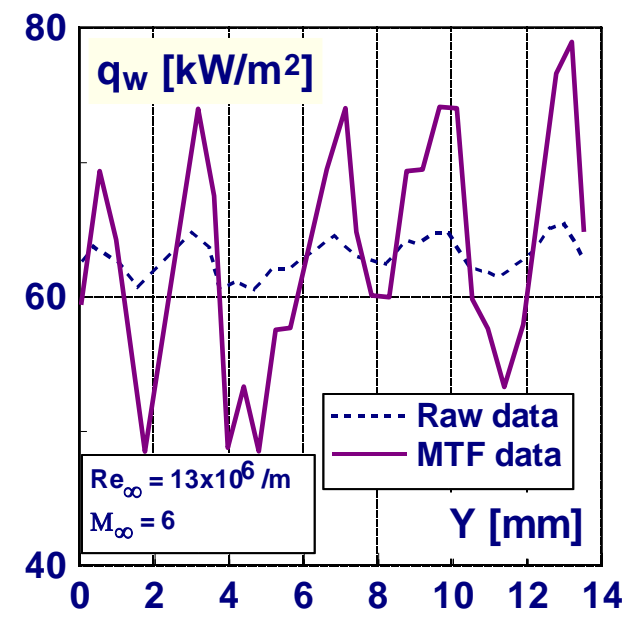

Fig. 3. Spanwise distribution of the heat flux.

A second problem of flows at high enthalpy is the heating of a plate by a flame. This case has been studied by Reulet \& Millan (2004) at the DMAE laboratory of ONERA in Toulouse. The objective is the determination of the instantaneous mapping of the heat flux on a flat metal surface impinged by a propane flame at $1600^{\circ} \mathrm{C}$. The experimental set-up is presented in Fig. 4a. The target is a steel plate, $3 \mathrm{~mm}$ thick. The stand-off distance between the nozzle and the plate varies from 2.5 to 4 jet diameters. The thermal measurement is performed by means of an AGEMA 880 LWB camera, which scans the upper face of the target via a mirror tilted at $45^{\circ}$. Typical thermogram recorded during the transient heating is shown in Fig. 4b. The very good symmetry of the temperature field is noticed.

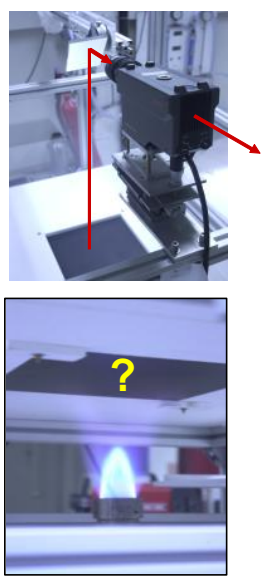

(a)Top and bottom view

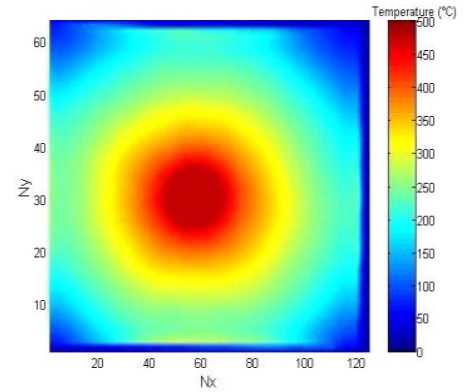

(b) Thermogram
Fig. 4. Impinging flame experiment.

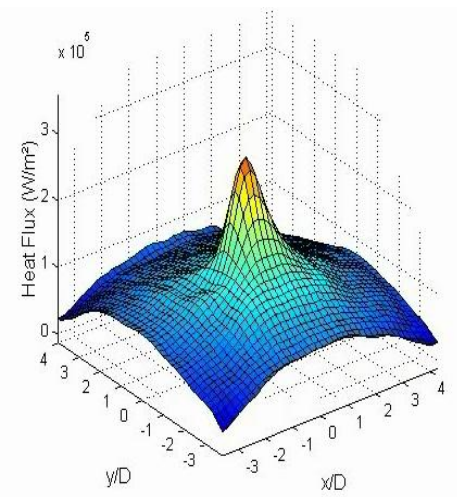

Fig. 5. Heat flux distribution at the flame impact

This instantaneous temperature distribution measured on the north surface of the plate is introduced as boundary conditions in a non-linear (thermo-physical properties depend on temperature) model of inverse method to retrieve the heat flux distribution on the south surface. An example of results is reproduced in Fig. 5.

\subsection{Gas Multijet Systems}

Multijet systems are encountered across a broad swathe of engineering processes where high heat and mass transfer rates are required. Typical examples are the annealing of metals, the tempering of glass sheets, the drying of paper and textiles, the cooling of turbine blades. The applications exemplified hereunder are the cooling of metal strips and the anti-icing of aircraft.

The objective is the determination of the convective heat transfer mapping at the impact of the multi jets (Buchlin, 2000).

Figure 6 provides a view of the experimental facility simulating at scale $1 / 3$ a unit of strip cooling and implemented with IRTh system. The set-up is composed of a plenum feeding an array of slot nozzles, the two at the centre being slightly tilted. The air jets impact a vertical flat plate, $1.7 \mathrm{~m}$ high and $0.27 \mathrm{~m}$ wide, made of an epoxy layer of $1 \mathrm{~mm}$ thick. The target is blanketed by a very thin cupper foil, $40 \mu \mathrm{m}$ thick, machined to form an electrical resistance. This Joule heating technique yields constant heat flux in time and uniform in space.

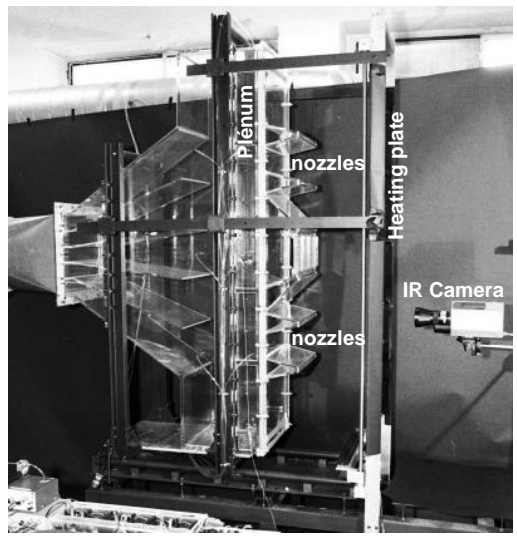

Fig. 6. Set-up of array of slot jets 
The rear face of the plate is black painted and scanned by a ThermaCAM ${ }^{\circledR}$ SC3000 camera. The camera is mounted on a vertical displacement mechanism to allow the measurement of the temperature field on all the height of the strip. The steady state data are processed by means of Eq. (7) to retrieve the distribution of the convective heat transfer coefficient.

The thermogram in Fig. 7a shows that the temperature field is essentially one-dimensional. That leads to consider only the thermal variation along the longitudinal direction Ox. A typical axial profile of the Nusselt number $\left(N u=h . S / k_{a}\right)$ based on the hydraulic diameter of the slot nozzle, $S$, is plotted in Fig. $7 \mathrm{~b}$. It is compared to a 3D CFD simulation performed with the Fluent code. The overall agreement found hints at the good modeling of such a complex flow topology.

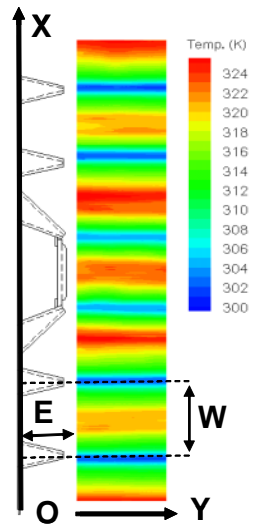

(a)

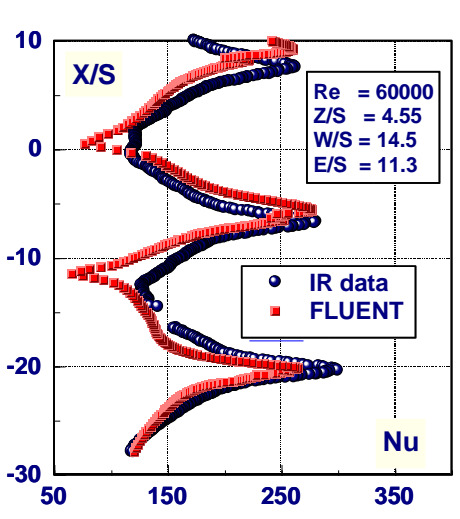

(b)
Fig. 7. Nu profile for array of slot nozzles

Multi jets are also operating in thermal anti-icing systems of wing, tail and engine inlet of airplanes. The principle is illustrated in Fig. 8; that consists in taking hot air from the engine circuit and blowing it in the front bay through a piccolo tube drilled of several round holes. The hot jets issuing from the piccolo tube warm up the leading edge avoiding or suppressing any accretion of ice when the airplane is flying in severe atmospheric conditions. The energetic optimization of such system relies upon the good knowledge of the heat exchange involved all the more so since the air mass flow drawn off from the engine is fairly limited.

The experimental approach undertaken at the VKI involves a full-scale model of the leading edge section of the Embraer ERJ 145 and Airbus 380. Photography of the test bench is proposed in Fig. 9. To be in a position to apply the active IRTh method, the skin of the model is made of a thin heating foil with uniform heat flux and black painted on the external face, which is scanned by the IR camera. Several titanium piccolo tube designs are tested. The impinging air jets are maintained at ambient temperature.

To investigate the convective heat transfer on all the surface of the strongly curved leading edge, the IR camera is positioned successively on different supports mounted on an arch structure shown in Fig. 9. The thermograms obtained in this way are patched together to reconstruct finally the complete thermal field.

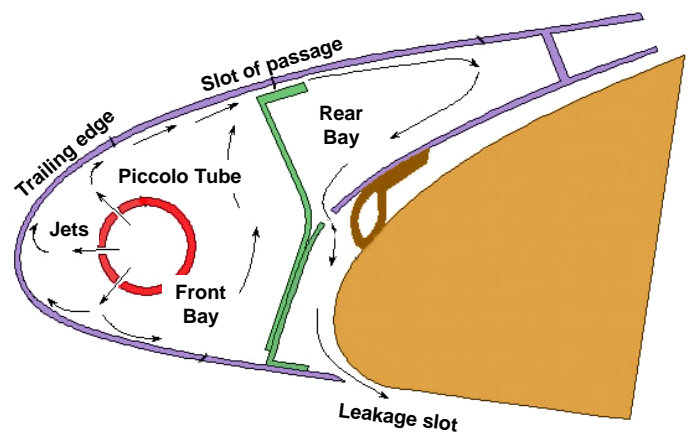

Fig. 8. Principle of the thermal anti-icing.

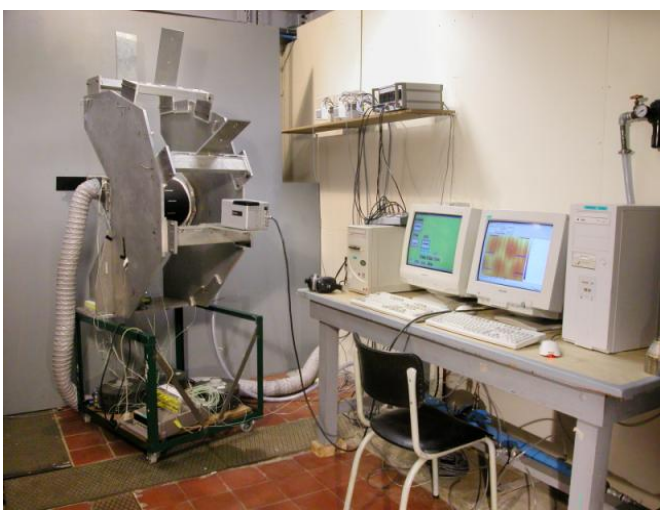

Fig. 9. Test bench for the thermal anti-icing experiments.

As an example Fig. 10a shows three thermograms, which once combined lead, after application of Eq. (7), to the mapping of the convective heat transfer coefficient (here dimensionless).
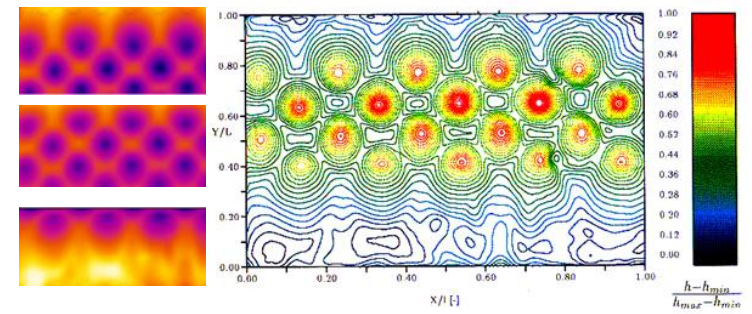

Fig. 10. Results for array of round jets.

One strives to compare the experimental data to CFD simulation performed with the code Fluent. The comparison relies on the mean Nusselt number, $\langle N u\rangle$, of a representative cell. This cell is defined by the jetto-jet chordwise pitch, $L_{c}$, and spanwise pitch, $L_{e}$, as explained in Fig. 11a where a 3D numerical simulation is shown. Figure $11 \mathrm{~b}$ confirms the good agreement between experiments and numerical simulation and allows proposing with confidence a $\langle N u\rangle$ correlation including the effect of the jet Reynolds number and a geometrical cell parameter $G o$ :

$G o=n_{j}^{a}\left[\frac{L_{c}}{d}\right]^{-a}\left[\frac{L_{e}}{d}\right]^{-b}\left[\frac{Z}{d}\right]^{-c}$

Where $n_{i}$ is the number of jet raws and $Z$ the shortest 
stand-off distance between the piccolo tube and the leading edge skin.

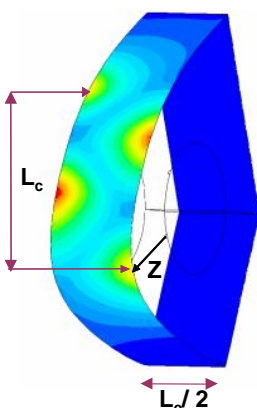

(a)

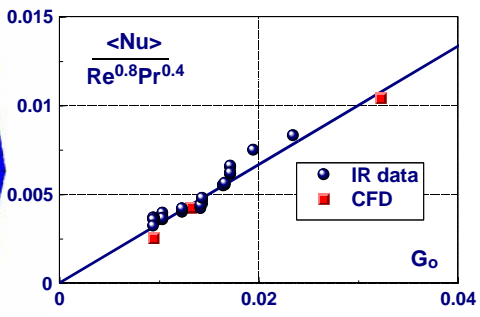

(b)
Fig. 11. Results for round multijets.

\subsection{The Turbulator Ribs}

Internal cooling of turbine blades as well as of highpower electronic components often requires the presence of turbulence promoters to enhance significantly the heat exchange. The ribbed channel is one of these devices. In such a configuration, the walls of the duct are fitted with prismatic cylinders, perpendicular to the flow or with a slight incidence. Figure 12 shows a LES simulation (Vass et al., 2007) of this type of flow. It emphasizes the net effect of these turbulators, which yield numerous turbulent macro structures (in color in the figure), which will promote the convective heat transfer.

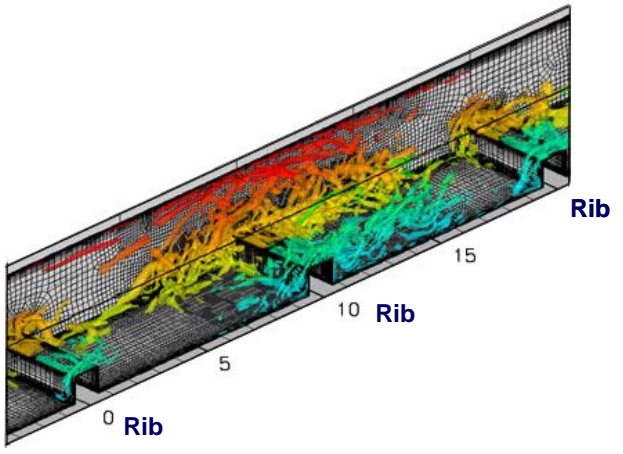

Fig. 12. Flow in ribbed channel.

The active IRTh method applies readily to such a geometrical layout. Figure 13a shows an example of an experimental setup used to study these promoters of turbulence.

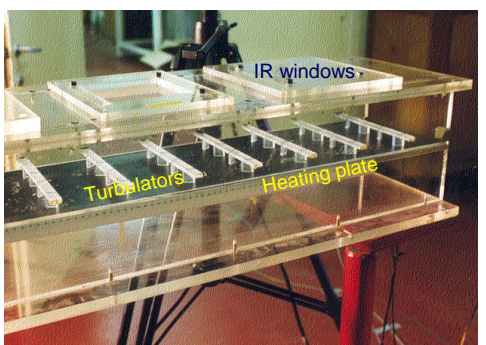

(a)

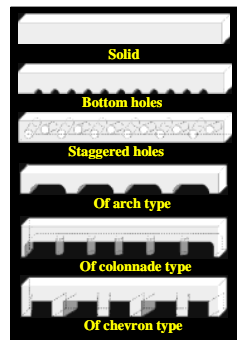

(b)
Fig. 13. Ribbed channel setup
The ribs are machined within a poor thermal conductor material. They are set on a flat plate of uniform heat flux (Joule effect with thermofoil technique). The area between ribs is successively analyzed with an AGEMA Thermovision 900 camera scanning via a $45^{\circ}$ mirror through IR windows made of thin polyester film stretched on frames. The study puts the emphasis on the concept of the perforated rib (Buchlin, 2002). The different types of perforations to be tested are sketched in Fig. 13b. The turbulators are characterized by the area porosity, $\beta$, which varies from 0 (solid rib) to 0.69 .

From a general point of view the heat transfer distribution between two successive ribs evolves along the channel to reach a developed state after the $5^{\text {th }}$ or $6^{\text {th }}$ promoter. Figure 14 gives a typical example of such evolution in the case of a channel with 7 perforated ribs of chevron type. In Fig. 14 the convective heat transfer coefficient is normalized by the value that would be obtained in smooth channel. The Reynolds number, based on the channel height and the free stream velocity, $U_{e}$, is 32000 and the turbulator pitch, $P$, is 5 times their height, $H$.

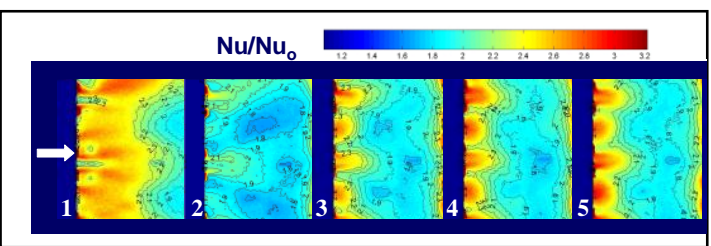

Fig. 14. Aerothermal development in channel fitted with perorated ribs of chevron type: $R e=32000, \beta=0.533, P / H=5$.

The turbulator performance can be expressed in terms of the mean Nusselt number, $\langle N u\rangle$, calcu;lated on the area between two successive ribs once the flow is developed. Figure 15 shows that the perforation of the rib brings an improvement, which can be more or less marked according to the design of the flow passages. Figure 15 points also out that the perforated rib of chevron type appears as a promising design.

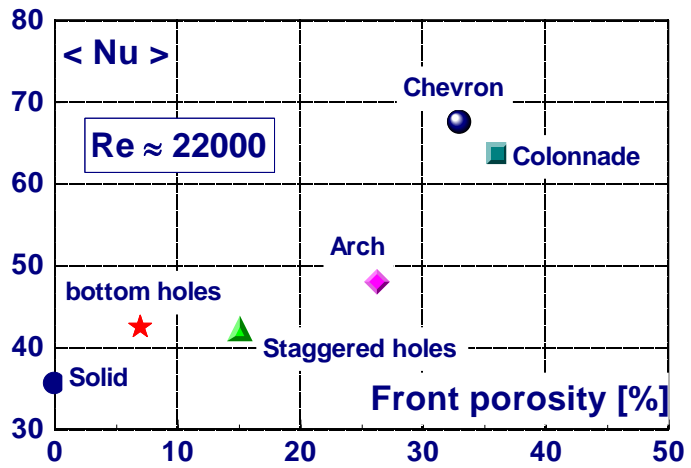

Fig. 15. Mean Nusselt number of perforated ribs.

It can be anticipated that perforated ribs will generate less pressure drop than solid ribs of same size. Therefore it is worthwhile seeking for an optimum of front porosity. The exercise is conducted with the perforated ribs of chevron type. The results are plotted in Fig. 16. 


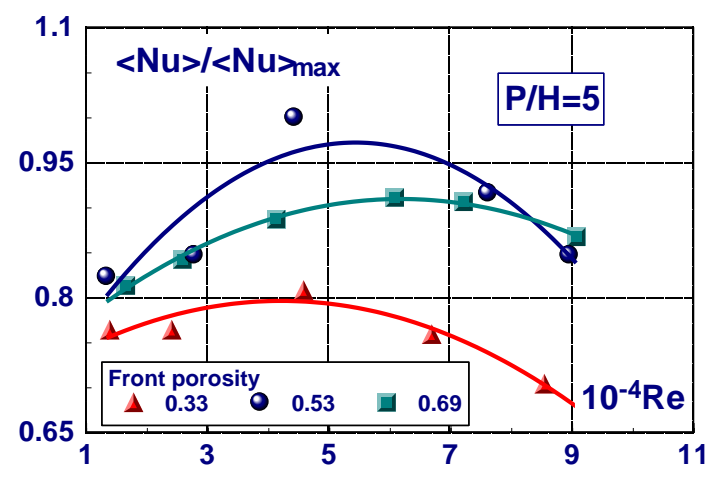

Fig. 16. Normalized mean Nusselt number of chevron type ribs versus Reynolds number.

At high velocity, the aeraulics resistance due to the rib permeability becomes too important; then air flow rate through the rib and subsequently the thermal efficacy decline.

\subsection{Fibre Cooling}

In polymer-fibre spinning process the careful control of the yarn quenching has a strong bearing on the final quality of the product. It is essential to know precisely the laws of heat exchange between the fibres, generally in motion, and the surrounding gas.

In such a problematic, the active IRTh method appears to be a useful tool.

An experimental study of fibre cooling has been carried out at the VKI on the setup sketched in Fig. 17 (Buchlin, 1998).

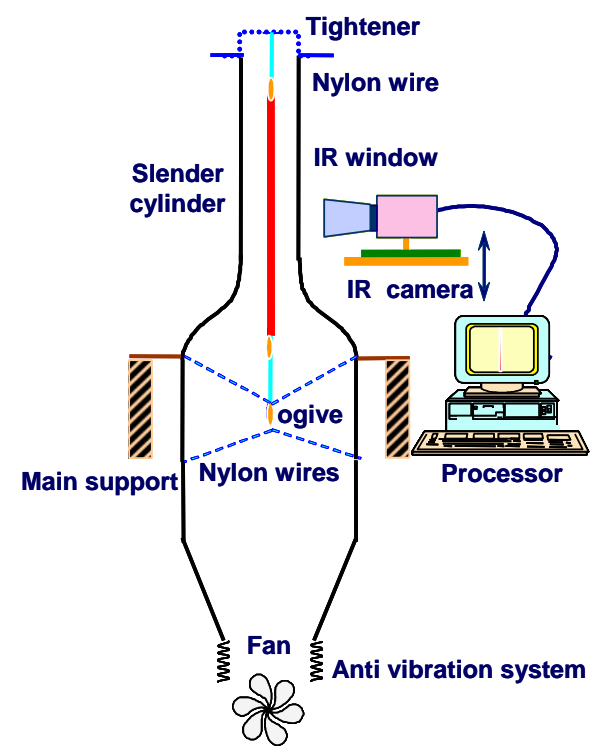

Fig. 17. Test setup for fibre cooling

The fibre is simulated by a slender cylinder, the radius of which can vary from $0.5 \mathrm{~mm}$ to $3.2 \mathrm{~mm}$. It is heated by Joule effect up to a maximum heat flux of 10000 $\mathrm{W} / \mathrm{m} 2$. The wire is taut along the axis of a wind tunnel; the test section is $0.2 \times 0.2 \mathrm{~m}^{2}$ and $1.8 \mathrm{~m}$ long. The flow velocity of the free stream, $U_{e}$, may vary from $1 \mathrm{~m} / \mathrm{s}$ to
$12 \mathrm{~m} / \mathrm{s}$ with a turbulence level of $0.5 \%$. To study the fibre-to-fibre interaction, the facility can accommodate a setting of two parallel slender cylinders with a standoff distance, $d_{i}$, adjustable between 7 and 10 wire radii. Moreover, the fastening system allows wire incidence from $-1.5^{\circ}$ to $+1.5^{\circ}$. The slender cylinders are coated with a black paint of high emissivity (0.96). An AGEMA Thermovision 900 camera scans the wires through IR windows similar to those used during the perforated rib study (see previous section). The optics and positioning of the IR radiometer warrant a spatial resolution of $1 / 3 \mathrm{~mm}$ on the target, enough to obtain the temperature evolution along the slender cylinder with a good accuracy.

The results of the measurements performed at steady state are expressed in terms of the Nusselt number, $N u_{a}$, based of the cylinder radius, $a$, and the curvature parameter $\xi$ defined as follows:

$\xi=\sqrt{\frac{x \cdot v}{U_{e} \cdot a^{2}}}$

In Eq. (10) $x$ is the axial abscissa and $v$ the air kinematic viscosity. Figure 18, which gathers the results corresponding to the single-wire tests, emphasizes the effect of the wire incidence.

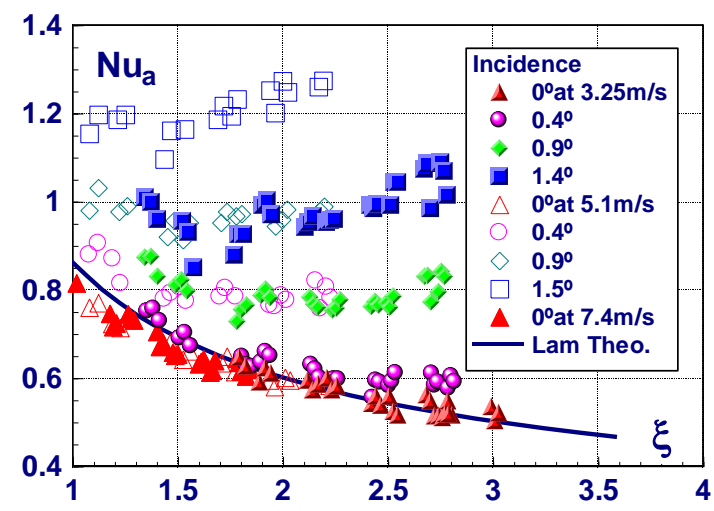

Fig. 18. Convective heat transfer for a single slender cylinder.

At zero incidence, the Nusselt number decreases as the curvature parameter increases; this is in very good agreement with the laminar theory (Richelle et al., 1995) as depicted in Fig. 18. Any small misalignment of the wire with respect of the free stream creates, however, a noticeable augmentation of heat transfer: an increase of a factor 2 can be readily attained for an incidence of $1.5^{\circ}$. This finding is fundamental when recalling that the fibres exit the extrusion dies and reach the bobbins with different angles. This would lead to very different cooling rates between fibre bundles and therefore very different mechanical behavior.

The effect of interaction between fibres on heat exchange is illustrated in Fig. 19 where the Nusselt number is plotted versus the curvature parameter for the case of two parallel wires without incidence and 7.6a apart. 
J.M. Buchlin / JAFM, Vol. 3, No. 1, pp. 55-62, 2010.

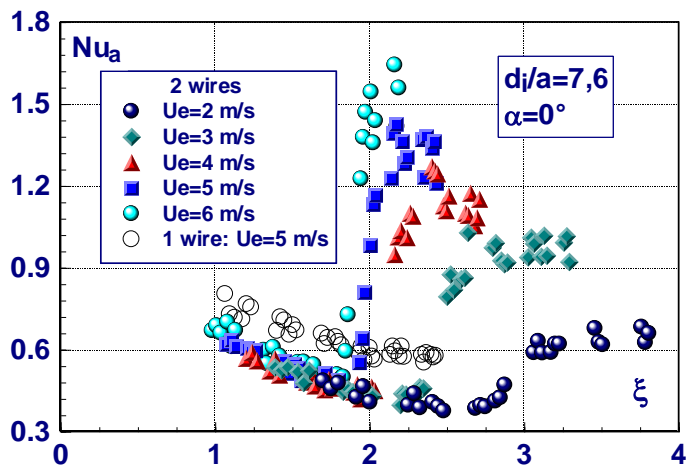

Fig. 19. Convective heat transfer for two parallel slender cylinders.

The proximity of the fibres affects the convective heat transfer since after a certain axial abscissa the evolution of the Nusselt number exhibits a sharp increase. This jump, which depends on the free stream velocity, is a clear indication of laminar to turbulent transition of the boundary layer. It turns out that in turbulent regime the curvature $\xi$ is not anymore the controlling parameter.

\section{CONCLUSION}

The infrared thermography is a thermometric technique of non-intrusive nature, which allows fast and detail measurement of the temperature distribution at the surface of a solid.

Its implementation relies upon the fundamental principles of the thermal radiation.

The most important features of IR radiometers are outlined to bring out the procedure to follow for making the IRTh a quantitative tool. In particular the criteria associated to thermal and spatial resolution have to be always kept in mind.

The potentiality offered by the IRTh to determine the mapping of the convective heat transfer coefficient in configurations of complex flow is demonstrated.

The passive and active IRTh methods are described and illustrated through several applications going from vehicle reentry in atmosphere, gas jet cooling, ribbed channels, anti/deicing of airplanes to fibre spinning.

\section{REFERENCES}

Buchlin, J.M. (2002). Convective heat transfer in a channel with perforated ribs (Transfert de chaleur par convection dans un canal muni de pontets perforés). International journal of thermal sciences ISSN 1290-0729, 41 (4), 332-340.

Buchlin, J.M. (2000). Convective Heat Transfer in impinging-Gas-Jet Systems. Aero-thermal Performance of Internal Cooling Systems in Turbomachines, VKI LS 2000-03.

Buchlin, J.M. (1998). Natural and forced convective heat transfer on slender cylinders. Revue Générale de Technique 37, 653-660.

Gaussorgues, G. (1989). La thermographie infrarouge principe, technologies, applications. Technique \& Documentation, Lavoisier.

Simeonides, G., J.P. Vermeulen, H.L. Boerrigter, and J.F. Wendt (1993). Quantitative heat transfer measurements in hypersonic wind tunnels by means of infrared thermography. IEEE Transactions on Aerospace and Electronic Systems 29(3), 878-893.

Simeonides, G., P. Van Lierde, S. Van der Stichele, D. Capriotti, and J.F. Wendt (1990). Infrared thermography in blowdown and intermittent hypersonic facilities. AIAA Journal of Thermophysics and Heat Transfer 4(2), 143-148.

Reulet, P. and P. Millan (2004). Convective Heat Transfer. Resolution of Three-Dimensional Unsteady Linear and Non Linear Inverse Problems using Infrared Observations. AVT/VKI Lecture Series on Heat Transfer and Inverse Analysis - Le transfert de chaleur et l'analyse inverse.

Richelle, E., R. Tasse, and M.L. Riethmuller (1995). Momentum and thermal boundary layer along a slender cylinder in axial flow. I.J. Heat \& Fluid Flow 16(2), 99-105.

Vass, P., P. Rambaud, T. Arts and C. Benocci (2007). Numerical investigation of flow and heat transfer in a ribbed square duct applying LES. 7th European Conference on Turbomachinery Fluid Dynamics and Thermodynamics, Athens, Greece. 